were successfully managed conservatively. There were no complications involving the distal ureteral stump. During follow-up (mean 33 months), however, two of the five patients aged $<1$ year (aged 6 months and 7 months) experienced loss of function of the remaining ipsilateral kidney moiety, despite ultrasound evidence of hilar blood flow. One of these patients showed pathologic evidence of dysplasia (hyaline cartilage nodules). The cause of the loss in renal function was unclear, but the authors note that retroperitoneal $\mathrm{CO}_{2}$ insufflation decreases renal blood flow, and speculate that infants aged $<1$ year might be particularly susceptible to peripheral renal vascular injury. Although the retroperitoneal approach can provide direct access to the renal hilum, transperitoneal surgery might be safer in this age-group.

Wallis et al. observed that their operative times decreased markedly with time. They call for prospective, longitudinal studies that compare the outcomes of patients who undergo transperitoneal, retroperitoneal and open procedures. Such studies should assess postoperative renal function with dimercaptosuccinic acid scans, and might elucidate potential causes of these reported complications.

Original article Wallis MC et al. (2006) Outcome analysis of retroperitoneal laparoscopic heminephrectomy in children. J Urol 175: 2277-2282

\section{Support for oral kinase inhibitor therapy in cytokine-refractory metastatic RCC}

Two multicenter trials carried out in the US have demonstrated the efficacy of the oral kinase inhibitors sunitinib and sorafenib in patients with metastatic, cytokine-refractory renal cell carcinoma (RCC). Only $15 \%$ of RCC patients typically respond to cytokines, so effective therapies are urgently needed. Sunitinib and sorafenib are thought to inhibit growth of RCC tumors via inhibition of several tyrosine protein kinases, including vascular endothelial growth factor and platelet-derived growth factors; RCC hypervascularity is associated with overexpression of these proangiogenic growth factors.

Motzer et al.'s open-label, multicenter trial involved 105 patients with confirmed metastatic, cytokine-refractory clear-cell RCC. All patients received repeated 6 -week treatment cycles comprising oral sunitinib $50 \mathrm{mg}$ daily for 4 weeks, followed by 2 weeks off treatment. In this study, 36 patients had a partial response to treatment and 30 patients had stable disease for at least 3 months. Median progression-free survival was 8.3 months-considerably longer than expected (2-4 months). When the study was published, median overall survival had not been reached, and the 6-month survival rate was $79 \%$.

Ratain et al.'s phase II study was designed to show whether sorafenib inhibits growth of various metastatic, treatment-refractory solid tumors. After the introductory 12 weeks of sorafenib, however, many patients with RCC showed tumor regression, and the remainder of the study focused on these patients. The authors used a controversial randomized discontinuation protocol to assess the clinical efficacy of sorafenib while minimizing the use of placebo: all 202 patients with metastatic, cytokine-refractory RCC initially received openlabel sorafenib $400 \mathrm{mg}$ twice daily for 12 weeks. Patients whose tumors increased in size by $\geq 25 \%$ discontinued sorafenib, while those whose tumors shrank by $\geq 25 \%(n=73)$ continued taking sorafenib, for a further 12 weeks. Patients with stable disease $( \pm 25 \%$ change in tumor size) were randomly allocated to receive either sorafenib $(n=32)$ or placebo $(n=33)$ for an additional, blinded 12 weeks. Patients whose tumors progressed during the second 12-week period were unblinded, and, if receiving placebo, were reallocated to sorafenib $(n=28)$ until further progression. Median progression-free survival after 24 weeks was much longer in the patients who took sorafenib than those who took placebo (24 weeks, compared with 6 weeks). Ratain et al. claim that this study design can efficiently assess treatments that have a primary clinical benefit of disease stabilization.

Sunitinib and sorafenib were reasonably well tolerated. Adverse effects, including fatigue, diarrhea, gastrointestinal symptoms, stomatitis, skin rashes and/or desquamation, hand-foot syndrome and hypertension were common, although generally mild $(11 \%$ and $9 \%$ of patients, respectively, discontinued therapy because of adverse effects; no patients died from treatment-related toxicity). Ratain and colleagues suggest that patients given sorafenib should also be prescribed antihypertensive medications. 
The oral kinase inhibitors show considerable promise for extending the survival of patients with RCC. Motzer et al. recently presented results that favor sunitinib, from their phase III trial of interferon a versus sunitinib as first-line therapy for metastatic RCC, at the American Society of Clinical Oncology June 2006 plenary session.

Original articles Motzer RJ et al. (2006) Sunitinib in patients with metastatic renal cell carcinoma. JAMA 295: 2516-2524 Ratain MJ et al. Phase II placebo-controlled randomized discontinuation trial of sorafenib in patients with metastatic renal cell carcinoma. J Clin Oncol 24: 2505-2512

\section{Accuracy of depression evaluation in hemodialysis patients}

Patients with end-stage renal disease on chronic hemodialysis often experience symptoms of depression. Adverse effects of dialysis including sleep disturbance, fatigue, and appetite and weight changes, however, could lead to erroneous diagnoses of depression as measured by self-report depression scales. Hedayati et al. explored the prevalence of depression in a group of 98 patients on dialysis and assessed the accuracy of different self-report scales.

The physician-administered Structured Clinical Interview for Depression (SCID) scale indicated that $26.7 \%$ of the group had a depressive disorder, and $17.3 \%$ major depression. In comparison with this scale, the Beck Depression Inventory (BDI), Center for Epidemiological Study of Depression (CESD) and Cognitive Depression Inventory (CDI) self-report scales were poor diagnostic tools. The BDI and CESD performed better, however, when their cutoff points for a diagnosis of depression were raised beyond those validated in the general population-supporting the notion that self-report scales record additional somatic depressive symptoms in hemodialysis patients that are not true indicators of depression. In further analyses, the CESD was found to be more accurate than the BDI at identifying individuals with depression.

The authors conclude that at the proposed new cutoffs the BDI and CESD are sufficiently accurate to be used as screening tools; they suggest that these tools be used to evaluate dialysis patients for depression at approximately yearly intervals, with patients with positive results being formally assessed by a physician or referred for psychiatric assessment. They do not recommend the CDI for screening because of its low sensitivity.

Original article Hedayati SS et al. (2006) The predictive value of self-report scales compared with physician diagnosis of depression in hemodialysis patients. Kidney Int 69: 1662-1668

\section{Perineural invasion is not prognostic in prostate cancer treated with brachytherapy}

The importance of perineural invasion (PNI) as a prognostic indicator in patients with prostate cancer is controversial and the data on the prognostic significance of PNI in those patients receiving brachytherapy are sparse. Researchers from the Cleveland Clinic Foundation, $\mathrm{OH}$, USA have now reported that PNI does not predict biochemical failure in patients with prostate cancer treated with brachytherapy.

In this matched-pair analysis, all patients received brachytherapy of 144 Gy to the prostate. Cases (biochemical failure) were matched with controls (no biochemical failure) for clinical stage, Gleason score, prostate-specific antigen level, age, race, and androgen deprivation status. A total of 56 pairs were available for analysis. Median follow-up time between cases and controls was comparable (58.5 and 53.5 months, respectively). The number of patients with PNI did not differ significantly between the two groups. Although there was a slight trend towards increased biochemical failure in patients with PNI, Kaplan-Meier analysis revealed that the difference between PNI-positive and PNI-negative patients was not statistically significant $(P=0.4014)$.

Although this study was limited by sample size, the authors conclude that the data are consistent with other reports in the literature that PNI does not have prognostic value in patients with prostate cancer. They suggest that post-therapy external beam radiation for patients with $\mathrm{PNI}$ is, therefore, unnecessary.

Original article Weight CJ et al. (2006) Perineural invasion on prostate needle biopsy does not predict biochemical failure following brachytherapy for prostate cancer. Int $J$ Radiat Oncol Biol Phys 65: 347-350 